\title{
Supplementary material:
}

1) List of instruments characteristic

Table S1 Measurement description

\begin{tabular}{|c|c|c|c|}
\hline $\begin{array}{l}\text { Scattering Instrument, } \\
\text { Model number }\end{array}$ & $\begin{array}{c}\text { Scattering } \\
\text { wavelengths }(\mathrm{nm})^{1}\end{array}$ & Corrections & Manufacturer Info \\
\hline $\begin{array}{l}\text { TSI total/backscatter } \\
\text { nephelometer, } 3563\end{array}$ & $450,550,700$ & Anderson and Ogren [1998] & $\begin{array}{c}\text { TSI, Inc. } \\
\text { St. Paul, MN USA }\end{array}$ \\
\hline $\begin{array}{c}\text { Optec open-air } \\
\text { nephelometer, NGN-2 }\end{array}$ & 550 & none applied & $\begin{array}{c}\text { Optec, Inc. } \\
\text { Lowell, MI USA }\end{array}$ \\
\hline $\begin{array}{l}\text { Radiance Research } \\
\text { nephelometer, M903 }\end{array}$ & 532 & $\begin{array}{l}\text { none applied } \\
\text { could use A\&O corrections for } \\
\text { assumed SAE (Table } 4 b ?) \\
\text { but probably doesn't matter for } \\
\text { trends since constant }\end{array}$ & $\begin{array}{c}\text { Radiance Research } \\
\text { Seattle, WA, USA }\end{array}$ \\
\hline $\begin{array}{c}\text { Ecotech Aurora } 3000 \\
\text { nephelometer, ... }\end{array}$ & $450,525,630$ & $\begin{array}{c}\text { Anderson and Ogren [1998] } \\
\text { should use A\&O based on Müller } \\
\text { et al., } 2011\end{array}$ & $\begin{array}{c}\text { Ecotech } \\
\text { Melbourne, Australia }\end{array}$ \\
\hline $\begin{array}{l}\text { Absorption Instrument, } \\
\text { Model number }\end{array}$ & $\begin{array}{c}\text { Absorption } \\
\text { wavelengths }(\mathrm{nm})^{1}\end{array}$ & Corrections & Manufacturer Info \\
\hline $\begin{array}{c}\text { Aethalometer AE9, AE10, } \\
\text { AE16: white light } \\
\text { Aethalometer AE21: } 2 \\
\text { wavelengths } \\
\text { Aethalometer AE31 and } \\
\text { AE33: } 7 \text { wavelengths }\end{array}$ & $\begin{array}{c}\text { broadband centered } \\
\text { at } 840 \mathrm{~nm} \\
370 \text { and } 880 \mathrm{~nm} \\
370,470,520,590,660 \\
, 880,950 \mathrm{~nm}\end{array}$ & $\begin{array}{c}\text { Cref }=1.80 \\
\text { AE31: } C_{r e f}=3.5 \\
\text { AE33: } C_{r e f}=3.0\end{array}$ & $\begin{array}{l}\text { Magee Scientific, } \\
\text { Berkeley, CA USA }\end{array}$ \\
\hline $\begin{array}{l}\text { PSAP - } 1 \text { wavelength } \\
\text { PSAP - } 3 \text { wavelengths }\end{array}$ & $\begin{array}{c}565^{\text {psap }} \\
467,530,660^{\text {psap }}\end{array}$ & $\begin{array}{l}\text { Bond et al. [1999] } \\
\text { Ogren [2010] }\end{array}$ & $\begin{array}{l}\text { Radiance Research, } \\
\text { Seattle, WA USA }\end{array}$ \\
\hline MAAP & 637 & none applied & $\begin{array}{l}\text { Thermo Scientific } \\
\text { Franklin, MA USA }\end{array}$ \\
\hline CLAP 3w & $467,528,652$ & $\begin{array}{l}\text { Bond et al. [1999] } \\
\text { Ogren [2010] }\end{array}$ & $\begin{array}{c}\text { NOAA } \\
\text { Boulder, CO USA }\end{array}$ \\
\hline
\end{tabular}

${ }^{1}$ Reported by manufacturer; psap In this work we use PSAP data adjusted to $550 \mathrm{~nm}$, based on Bond et al. [1999] for 1- wavelength instrument or Ogren, [2010] for 3-wavelength. 
2) Present-day MK results not shown in the paper

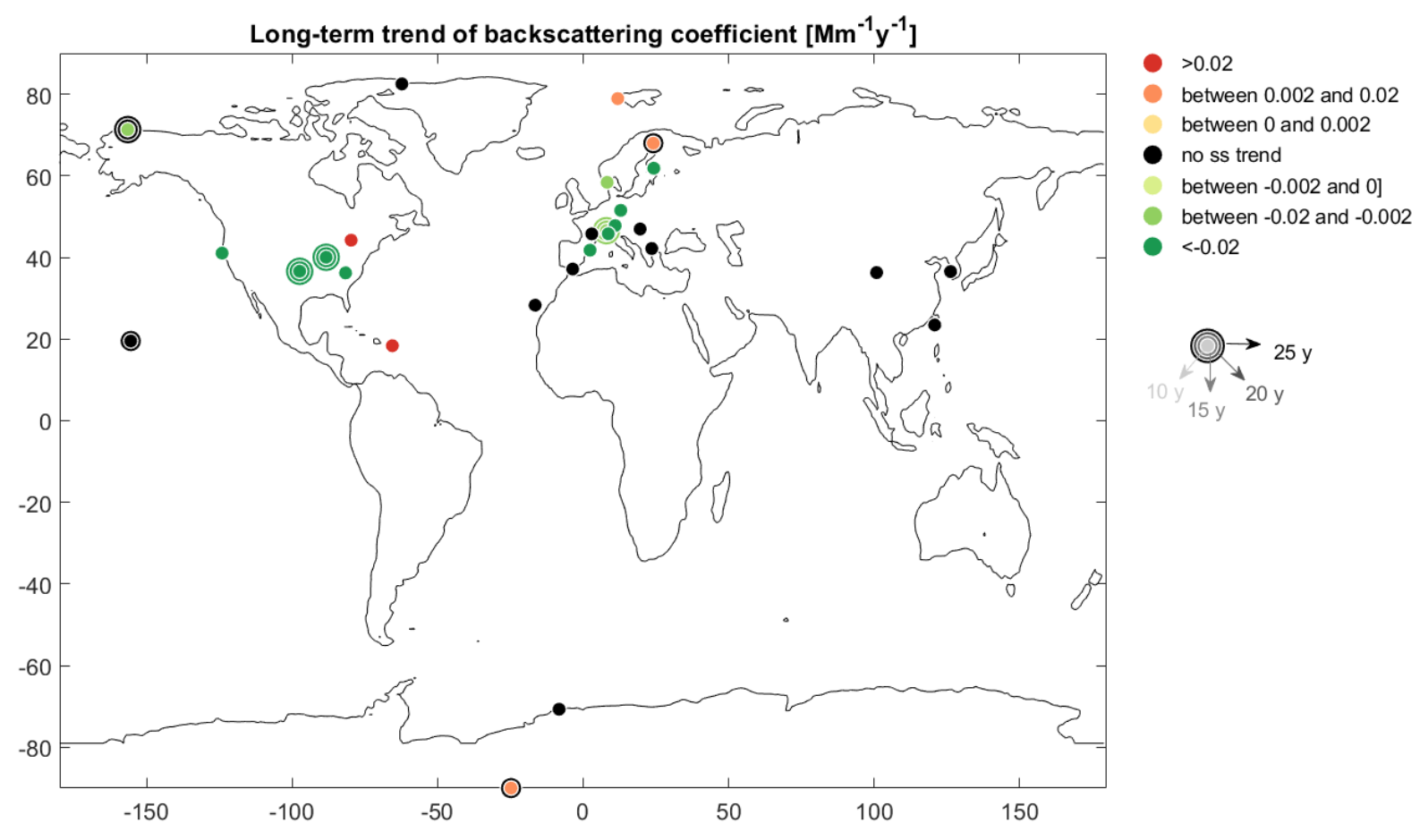

Fig. S1: MK trends results for the backscattering coefficient. Black symbols correspond to stations with no significant trends. Green and orange symbols correspond to statistically significant negative and positive trends, respectively, the magnitude of the trends (slope) being given by the colors as stipulated in the legend. The size of the circles are proportional to the length of the data sets with the central dots representing the present-day 10 years trend ending in 2016, 2017 or 2018 . If possible, trends for longer time periods were calculated and the larger circles denote the trends for 15 y to $40 \mathrm{y}$ in 5 year increments. 


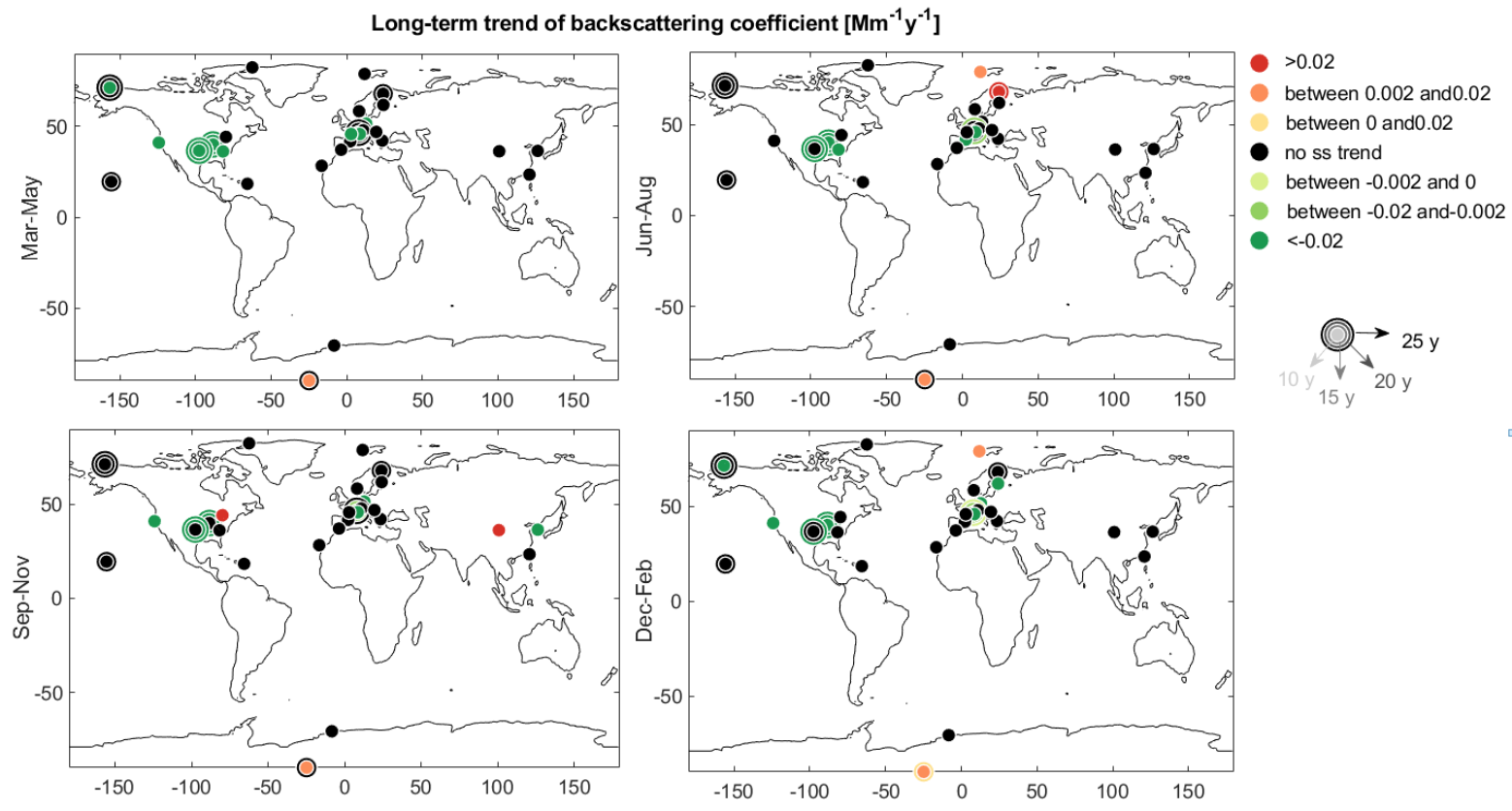

Fig. S2: Seasonal results of the MK trend of the backscattering coefficient. Other details same as Fig. $\mathrm{S} 1$.

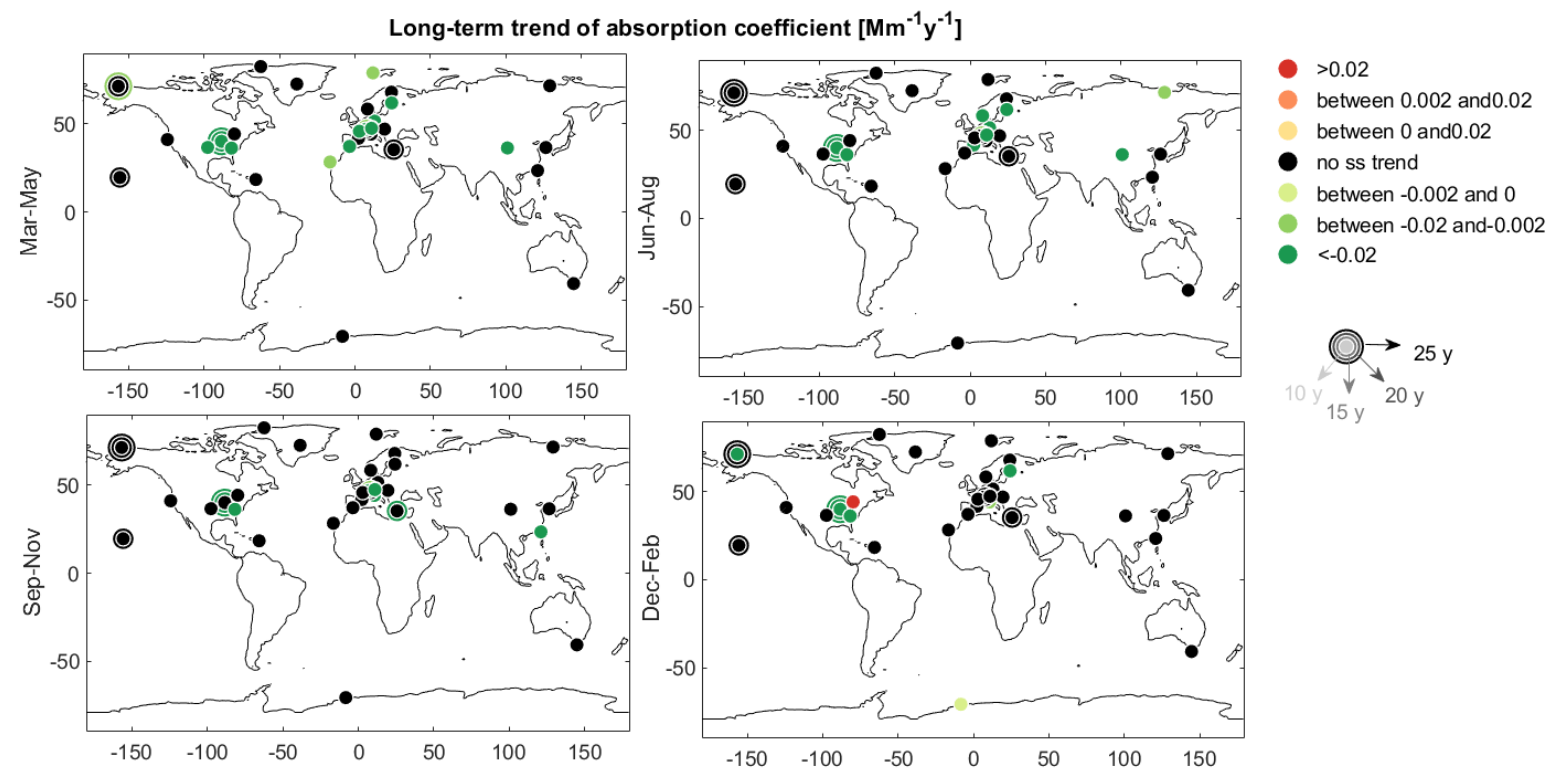

Fig. S3: Seasonal results of the MK trend of the absorption coefficient. Other details same as Fig. S1. 


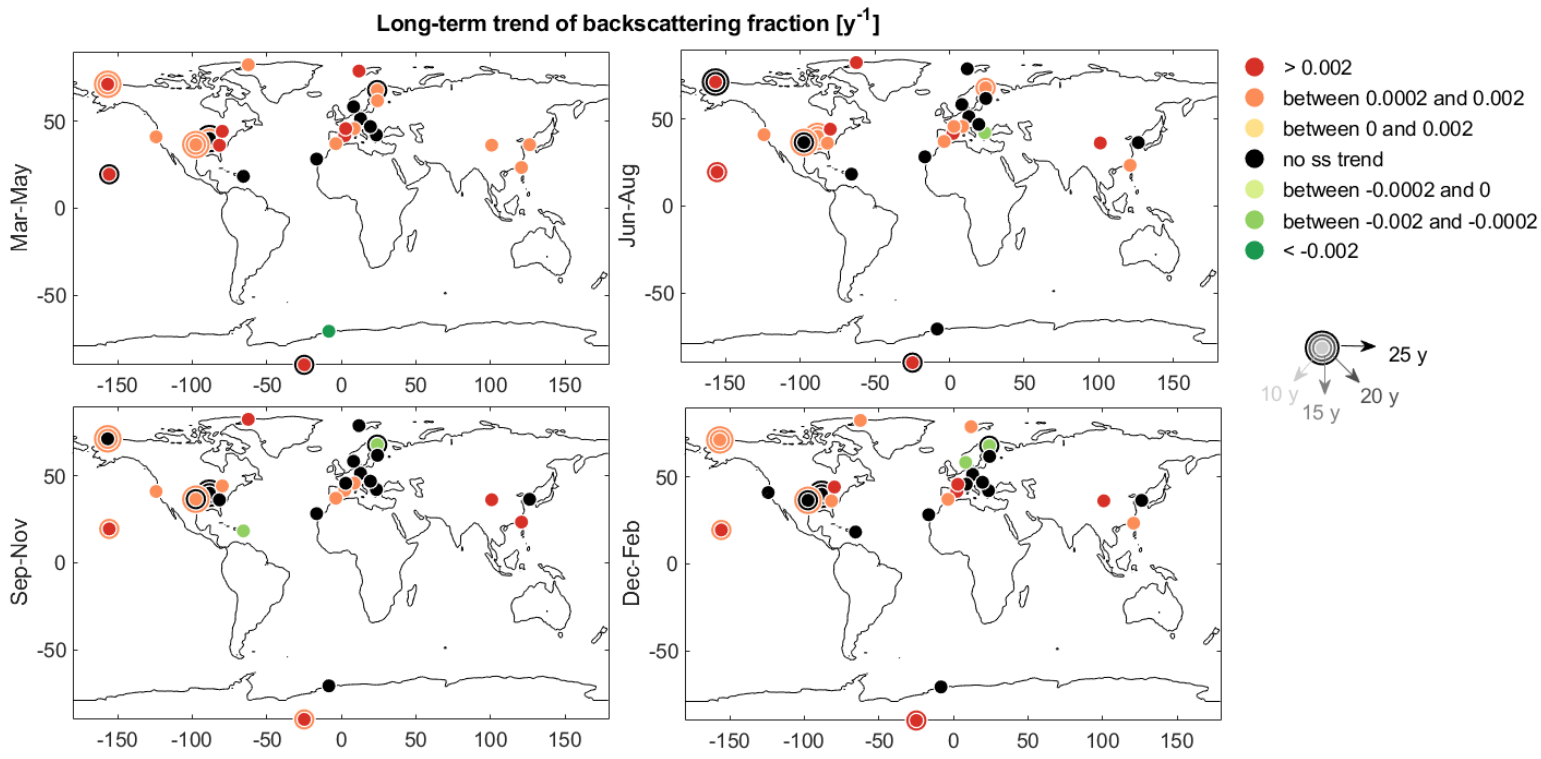

Fig. S4: Seasonal results of the MK trend of the backscattering fraction. Other details same as Fig. S1.
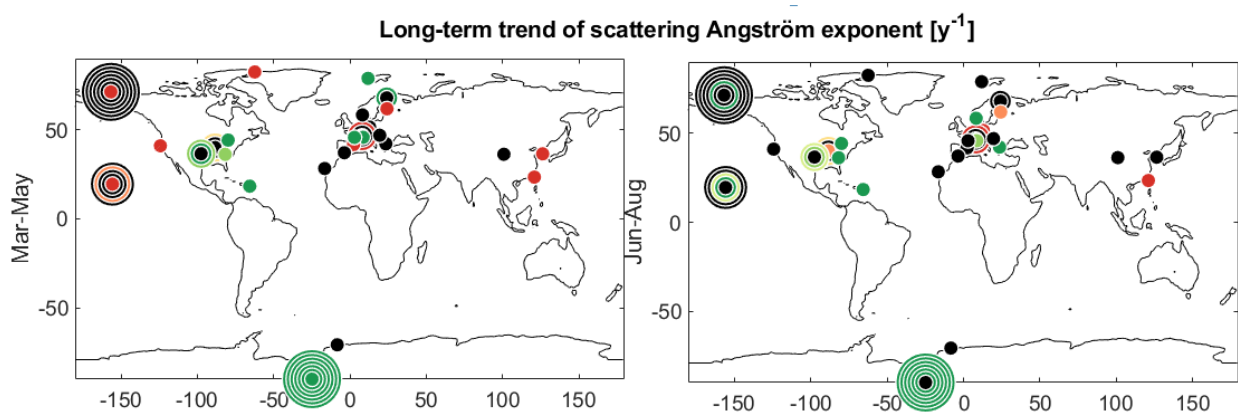

$>0.01$

between 0.005 and 0.01

between 0 and 0.01

- no ss trend

between -0.005 and 0

between -0.01 and -0.005

$<-0.01$
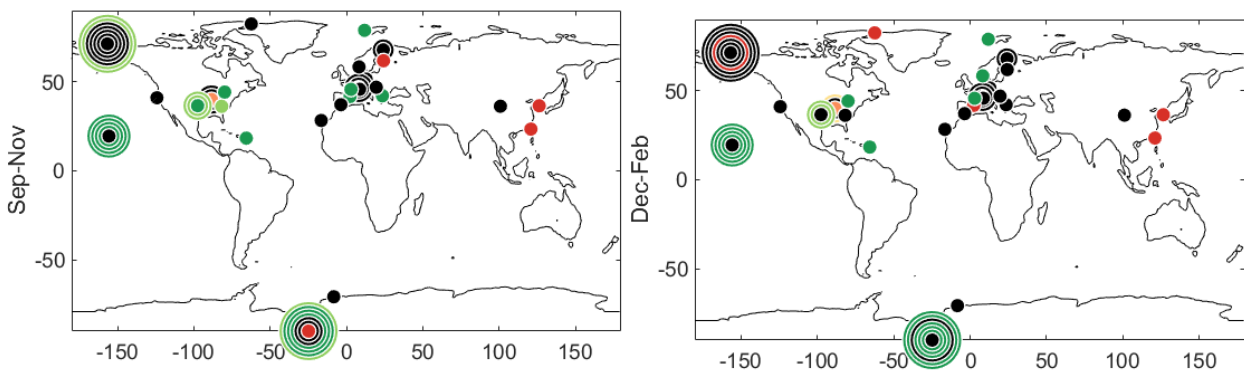

Fig. S5: Seasonal results of the MK trend of the scattering Angström exponent. Other details same as Fig. S1. 


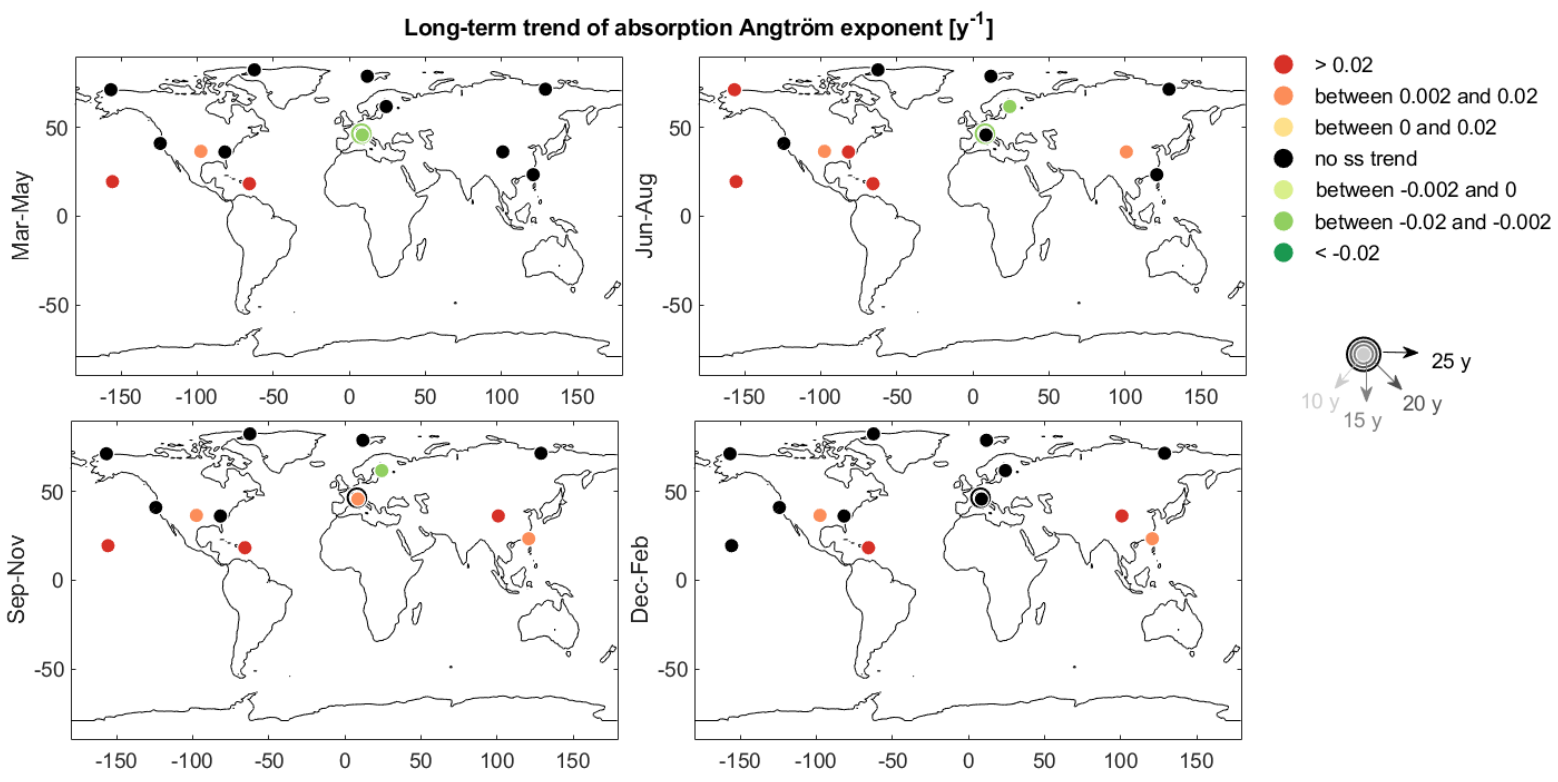

Fig. S6: Seasonal results of the MK trend of the absorption Angström exponent. Other details same as Fig. S1. 
3) Present-day trend results for MK, LMS and GLS/ARB methods in \%/y

Table S1: Present -day results for MK in \%/y

\begin{tabular}{|c|c|c|c|c|c|c|c|c|c|c|c|c|c|c|c|c|c|c|c|c|}
\hline \multirow[t]{2}{*}{ Station } & \multicolumn{3}{|l|}{$\sigma_{\mathrm{sp}}$} & \multicolumn{3}{|l|}{$\sigma_{\text {bsp }}$} & \multicolumn{3}{|l|}{$\sigma_{\text {ap }}$} & \multicolumn{3}{|c|}{$\omega_{0}$} & \multicolumn{3}{|l|}{ b } & \multicolumn{3}{|l|}{ åsp } & \multicolumn{2}{|l|}{ åap } \\
\hline & $10 \mathrm{y}$ & $15 \mathrm{y}$ & $20 \mathrm{y}$ & $10 \mathrm{y}$ & $15 y$ & $20 \mathrm{y}$ & $10 y$ & $15 \mathrm{y}$ & $20 \mathrm{y}$ & $10 y$ & $15 \mathrm{y}$ & $20 \mathrm{y}$ & $10 \mathrm{y}$ & $15 \mathrm{y}$ & $20 \mathrm{y}$ & $10 \mathrm{y}$ & $15 \mathrm{y}$ & $20 \mathrm{y}$ & $10 \mathrm{y}$ & $15 \mathrm{y}$ \\
\hline \multicolumn{21}{|l|}{ Africa } \\
\hline IZO & -4.60 & & & -2.31 & & & -3.84 & & & $\begin{array}{c}-0.02 \\
\end{array}$ & & & 0.41 & & & 0.15 & -4.60 & & & \\
\hline \multicolumn{21}{|l|}{ Asia } \\
\hline AMY & -1.62 & & & -1.29 & & & -0.27 & & & -0.09 & & & 0.46 & & & 1.19 & 1.13 & & & \\
\hline LLN & -0.79 & & & -0.64 & & & -6.76 & & & 0.40 & & & 1.64 & & & 1.36 & & & 0.54 & \\
\hline WLG & -1.48 & & & 0.57 & & & -2.90 & & & 0.05 & & & 1.86 & & & 1.68 & & & 2.30 & \\
\hline \multicolumn{21}{|c|}{ Europe } \\
\hline BEO & -0.79 & & & 3.76 & & & & & & & & & -1.29 & & & -1.15 & & & & \\
\hline BIR & $\begin{array}{l}-1.99 \\
\end{array}$ & & & $\begin{array}{l}-2.03 \\
\end{array}$ & & & $\begin{array}{l}-2.96 \\
\end{array}$ & & & 0.11 & & & $\begin{array}{l}-0.32 \\
\end{array}$ & & & $\begin{array}{l}-1.36 \\
\end{array}$ & & & & \\
\hline$\overline{C M N}$ & & & & & & & \begin{tabular}{|l|}
-1.83 \\
\end{tabular} & & & & & & & & & & & & & \\
\hline FKL & & & & & & & 0.41 & -0.17 & & & & & & & & & & & & \\
\hline HPB & -2.80 & & & -2.11 & & & -4.00 & & & 0.17 & & & & & & & & & & \\
\hline HYY & $\begin{array}{l}-2.92 \\
\end{array}$ & & & -2.24 & & & -4.20 & & & 0.27 & & & 0.53 & & & 0.91 & 0.91 & & -0.51 & \\
\hline IPR & -7.30 & & & -6.71 & & & -1.85 & & & -0.95 & & & 0.75 & & & -0.50 & & & 0.29 & \\
\hline JFJ & -8.34 & -5.35 & -2.89 & -4.71 & -3.55 & -2.28 & $\begin{array}{l}-10.22 \\
\end{array}$ & -3.37 & & \begin{tabular}{|c|}
-0.31 \\
\end{tabular} & \begin{tabular}{c|}
-0.11 \\
\end{tabular} & & & & & -1.61 & -0.43 & 0.21 & -0.73 & -0.57 \\
\hline KPS & -0.12 & & & -0.12 & & & -0.50 & & & 0.19 & & & -0.28 & & & -0.14 & & & & \\
\hline MPZ & -5.63 & & & -5.48 & & & -5.98 & & & 0.02 & & & -0.06 & & & 0.70 & & & & \\
\hline MSY & -6.00 & & & -4.02 & & & -1.67 & & & -0.41 & & & 2.44 & & & 0.62 & & & & \\
\hline PAL & 2.76 & 0.56 & & 4.09 & 0.97 & & $\begin{array}{l}-3.86 \\
\end{array}$ & & & 0.25 & & & -0.32 & -0.31 & & 0.35 & -0.18 & & & \\
\hline PAY & & & & & & & -3.29 & & & & & & & & & & & & & \\
\hline PUY & -3.24 & & & -2.10 & & & \begin{tabular}{|l|}
-3.62 \\
\end{tabular} & & & 0.23 & & & 1.70 & & & -0.97 & & & & \\
\hline UGR & 1.31 & & & 1.66 & & & \begin{tabular}{|c|}
-0.44 \\
\end{tabular} & & & 0.11 & & & 1.03 & & & 0.65 & & & & \\
\hline ZSF & & & & & & & -11.79 & & & & & & & & & & & & & \\
\hline \multicolumn{21}{|c|}{ North America } \\
\hline ACA & -5.58 & -2.75 & -2.42 & & & & & & & & & & & & & & & & & \\
\hline APP & -4.29 & & & -3.28 & & & -5.36 & & & 0.10 & & & 1.34 & & & -0.47 & & & -0.06 & \\
\hline BND & -3.86 & -2.42 & -1.84 & -3.61 & -2.28 & $\begin{array}{c}-1.74 \\
\end{array}$ & $\begin{array}{l}-3.12 \\
\end{array}$ & -3.17 & -1.47 & -0.07 & -0.03 & -0.06 & 0.18 & 0.43 & 0.18 & 0.54 & 0.10 & 0.25 & & \\
\hline CPR & 2.93 & & & 2.59 & & & -2.63 & & & 0.12 & & & -0.34 & & & -6.34 & & & 7.57 & \\
\hline EGB & 0.63 & & & 2.52 & & & 3.20 & & & 0.01 & & & 2.29 & & & -1.71 & -1.43 & & & \\
\hline
\end{tabular}




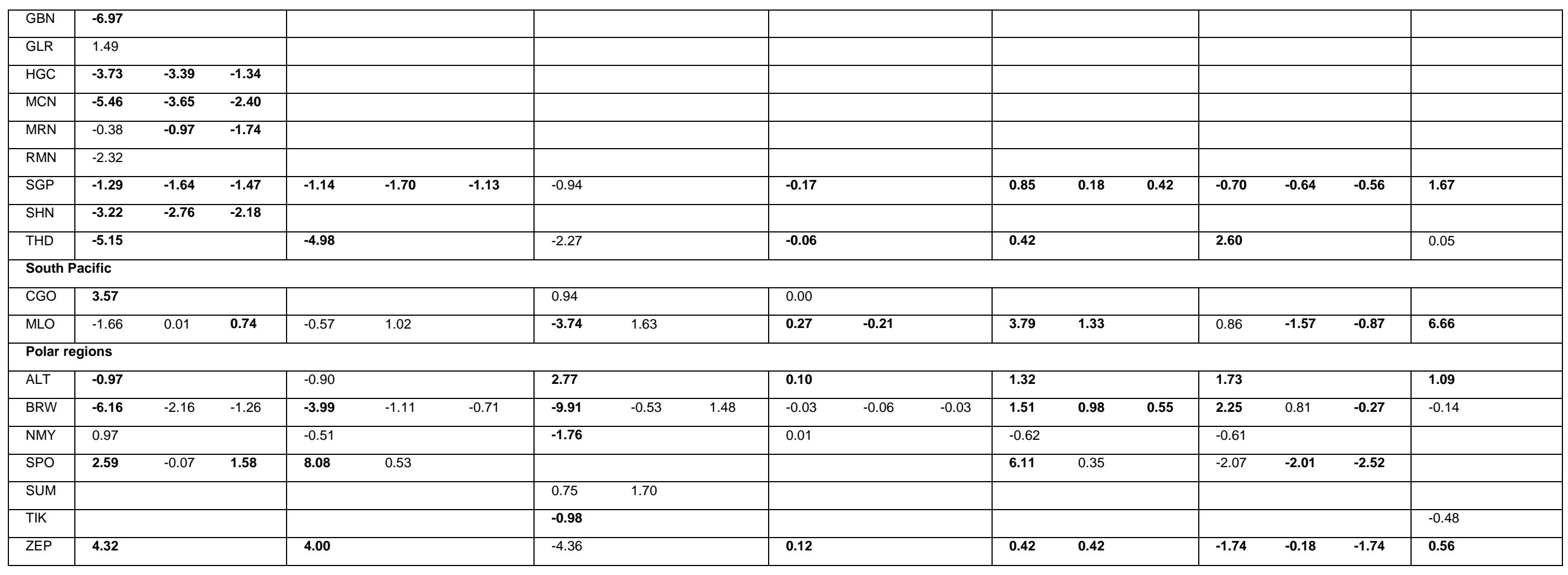

Table S2: Present -day results for GLS/ARB applied on daily median in \%/y

\begin{tabular}{|c|c|c|c|c|c|c|c|c|c|c|c|c|c|c|c|c|c|c|c|c|}
\hline \multirow[t]{2}{*}{ Station } & \multicolumn{3}{|l|}{$\sigma_{\mathrm{sp}}$} & \multicolumn{3}{|l|}{$\sigma_{b s p}$} & \multicolumn{3}{|l|}{$\sigma_{\mathrm{ap}}$} & \multicolumn{3}{|c|}{$\omega_{0}$} & \multicolumn{3}{|l|}{$b$} & \multicolumn{3}{|l|}{ åsp } & \multicolumn{2}{|l|}{ åap } \\
\hline & $10 \mathrm{y}$ & $15 \mathrm{y}$ & $20 \mathrm{y}$ & $10 \mathrm{y}$ & $15 \mathrm{y}$ & $20 \mathrm{y}$ & $10 \mathrm{y}$ & $15 \mathrm{y}$ & $20 \mathrm{y}$ & $10 \mathrm{y}$ & $15 \mathrm{y}$ & $20 \mathrm{y}$ & $10 \mathrm{y}$ & $15 \mathrm{y}$ & $20 \mathrm{y}$ & $10 \mathrm{y}$ & $15 \mathrm{y}$ & $20 \mathrm{y}$ & $10 y$ & $15 \mathrm{y}$ \\
\hline \multicolumn{21}{|l|}{ Africa } \\
\hline IZO & 11.30 & & & 11.76 & & & 0.48 & & & 0.10 & & & 0.45 & & & -2.04 & & & & \\
\hline \multicolumn{21}{|l|}{ Asia } \\
\hline AMY & -3.11 & & & -2.59 & & & -1.52 & & & -0.06 & & & 0.27 & & & 1.01 & 1.03 & & & \\
\hline LLN & -5.39 & & & -2.39 & & & -10.48 & & & 0.57 & & & 2.52 & & & 1.53 & & & 1.12 & \\
\hline WLG & -7.45 & & & -2.10 & & & -6.57 & & & 0.08 & & & 3.38 & & & 0.12 & & & 3.30 & \\
\hline \multicolumn{21}{|l|}{ Europe } \\
\hline $\mathrm{BEO}$ & -4.69 & & & 0.12 & & & & & & & & & -1.47 & & & -2.16 & & & & \\
\hline
\end{tabular}




\begin{tabular}{|c|c|c|c|c|c|c|c|c|c|c|c|c|c|c|c|c|c|c|c|}
\hline BIR & -6.49 & & & -6.09 & & & -16.84 & & & -0.04 & & & -0.30 & & & -2.35 & & & \\
\hline CMN & & & & & & & -7.80 & & & & & & & & & & & & \\
\hline FKL & & & & & & & 0.77 & -1.69 & & & & & & & & & & & \\
\hline HPB & -6.67 & & & -4.46 & & & -8.15 & & & 0.15 & & & & & & & & & \\
\hline HYY & -5.90 & & & -2.96 & & & -10.35 & & & 0.48 & & & 0.70 & & & 0.97 & 0.97 & & -0.63 \\
\hline IPR & -18.89 & & & -15.80 & & & -9.91 & & & -1.53 & & & -0.13 & & & -0.54 & & & 0.19 \\
\hline JFJ & -24.47 & $\begin{array}{l}- \\
12.29\end{array}$ & -6.60 & -12.56 & -7.61 & -5.37 & -14.23 & -7.76 & & -0.36 & -0.15 & & & & & -2.01 & -0.88 & -0.11 & $\begin{array}{ll}-1.20 & -0.53\end{array}$ \\
\hline KPS & -0.42 & & & -0.67 & & & -0.90 & & & 0.20 & & & -0.03 & & & -0.20 & & & \\
\hline $\begin{array}{l}\text { MPZ } \\
\end{array}$ & -7.60 & & & $\begin{array}{l}-5.68 \\
\end{array}$ & & & -9.25 & & & 0.12 & & & -0.38 & & & 0.15 & & & \\
\hline $\mathrm{MSY}$ & -12.14 & & & -6.18 & & & -3.18 & & & -0.44 & & & 4.60 & & & 2.08 & & & \\
\hline PAL & 10.59 & 1.44 & & 11.67 & 2.72 & & -5.55 & & & 0.55 & & & 0.96 & 0.83 & & 0.36 & -0.23 & & \\
\hline PAY & & & & & & & -4.04 & & & & & & & & & & & & \\
\hline PUY & -5.39 & & & -2.15 & & & -5.18 & & & 0.50 & & & 3.14 & & & -2.03 & & & \\
\hline UGR & -0.92 & & & 0.49 & & & -2.44 & & & 0.41 & & & 1.22 & & & 0.88 & & & \\
\hline ZSF & & & & & & & -20.45 & & & & & & & & & & & & \\
\hline \multicolumn{20}{|c|}{ North America } \\
\hline ACA & -9.41 & -7.62 & -5.49 & & & & & & & & & & & & & & & & \\
\hline APP & -5.92 & & & -3.51 & & & -6.35 & & & 0.11 & & & 1.50 & & & -0.68 & & & 1.52 \\
\hline BND & -6.54 & -4.67 & -3.06 & -5.36 & -3.41 & -2.48 & -3.70 & -4.57 & -2.04 & -0.13 & -0.02 & -0.01 & 0.38 & 0.54 & 0.15 & 0.43 & 0.03 & 0.22 & \\
\hline CPR & 2.57 & & & 2.35 & & & -5.37 & & & 0.23 & & & -0.27 & & & -9.80 & & & 8.05 \\
\hline EGB & -2.61 & & & 1.87 & & & 3.28 & & & -0.03 & & & 2.58 & & & -2.34 & -2.22 & & \\
\hline$\overline{\text { GBN }}$ & -5.84 & & & & & & & & & & & & & & & & & & \\
\hline GLR & 1.99 & & & & & & & & & & & & & & & & & & \\
\hline HGC & -7.48 & -5.49 & -2.91 & & & & & & & & & & & & & & & & \\
\hline $\mathrm{MCN}$ & -7.06 & -7.11 & -5.09 & & & & & & & & & & & & & & & & \\
\hline MRN & -0.55 & $\begin{array}{l}-1.04 \\
\end{array}$ & -1.95 & & & & & & & & & & & & & & & & \\
\hline RMN & -1.44 & & & & & & & & & & & & & & & & & & \\
\hline SGP & -3.40 & -3.61 & -3.09 & -2.87 & -3.13 & -2.06 & $\begin{array}{l}-1.38 \\
\end{array}$ & & & -0.12 & & & 0.88 & 0.30 & 0.57 & -1.03 & -0.93 & -0.72 & 2.44 \\
\hline SHN & -6.82 & -8.41 & -6.11 & & & & & & & & & & & & & & & & \\
\hline THD & -6.78 & & & -5.96 & & & -10.62 & & & -0.06 & & & 0.94 & & & 3.01 & & & 0.50 \\
\hline \multicolumn{20}{|c|}{ South Pacific } \\
\hline CGO & 3.98 & & & & & & 6.49 & & & 0.06 & & & & & & & & & \\
\hline MLO & -15.20 & -0.77 & 2.59 & -5.64 & 0.34 & & -18.98 & 2.84 & & 0.31 & -0.37 & & 3.96 & 0.86 & & 0.03 & -2.15 & -1.84 & 9.23 \\
\hline \multicolumn{20}{|c|}{ Polar regions } \\
\hline ALT & -5.36 & & & -3.39 & & & -9.06 & & & 0.23 & & & 3.31 & & & 1.08 & & & 1.07 \\
\hline BRW & -7.13 & -1.44 & -0.65 & -3.60 & -0.08 & 0.12 & -11.33 & -0.94 & -3.00 & 0.14 & 0.12 & 0.03 & 5.00 & -2.31 & 0.05 & 1.82 & -1.03 & -1.40 & 1.15 \\
\hline
\end{tabular}




\begin{tabular}{|c|c|c|c|c|c|c|c|c|c|c|c|c|c|c|}
\hline NMY & 42.10 & & & 295. & & -5.07 & & 0.02 & -1.38 & & -1.10 & & & \\
\hline SPO & & -2.44 & 1.24 & & 0.06 & & & & 7.84 & 1.12 & -1.77 & -1.62 & -1.96 & \\
\hline SUM & & & & & & -5.20 & 0.22 & & & & & & & \\
\hline TIK & & & & & & 0.25 & & & & & & & & -0.38 \\
\hline ZEP & 6.14 & & & 7.68 & & 0.78 & & 0.13 & 1.67 & 1.67 & -1.94 & -0.86 & -1.94 & 0.62 \\
\hline
\end{tabular}

Table S3: Present -day results for LMS applied on the logarithm of monthly median in \%/period

\begin{tabular}{|c|c|c|c|c|c|c|c|c|c|c|c|c|c|c|c|c|c|c|c|c|}
\hline \multirow[t]{2}{*}{ Station } & \multicolumn{3}{|l|}{$\sigma_{\mathrm{sp}}$} & \multicolumn{3}{|l|}{$\sigma_{b s p}$} & \multicolumn{3}{|l|}{$\sigma_{\text {ap }}$} & \multicolumn{3}{|c|}{$\omega_{0}$} & \multicolumn{3}{|l|}{ b } & \multicolumn{3}{|l|}{ åsp } & \multicolumn{2}{|l|}{ åap } \\
\hline & $10 y$ & $15 \mathrm{y}$ & $20 \mathrm{y}$ & $10 \mathrm{y}$ & $15 \mathrm{y}$ & $20 y$ & $10 y$ & $15 \mathrm{y}$ & $20 \mathrm{y}$ & $10 \mathrm{y}$ & $15 y$ & $20 y$ & $10 y$ & $15 \mathrm{y}$ & $20 \mathrm{y}$ & $10 y$ & $15 \mathrm{y}$ & $20 y$ & $10 y$ & $15 \mathrm{y}$ \\
\hline \multicolumn{21}{|l|}{ Africa } \\
\hline IZO & $\begin{array}{l}-0.92 \\
\end{array}$ & & & 0.71 & & & -2.95 & & & $\begin{array}{l}-0.10 \\
\end{array}$ & & & 0.07 & & & -10.75 & & & & \\
\hline \multicolumn{21}{|l|}{ Asia } \\
\hline AMY & -0.47 & & & -0.89 & & & -1.16 & & & -1.46 & & & 0.08 & & & 2.32 & 2.58 & & & \\
\hline LLN & $\begin{array}{l}-1.10 \\
\end{array}$ & & & $\begin{array}{l}-2.71 \\
\end{array}$ & & & -76.35 & & & 5.72 & & & 0.82 & & & 3.04 & & & 11.88 & \\
\hline WLG & \begin{tabular}{|l|}
-0.88 \\
\end{tabular} & & & 0.12 & & & $\begin{array}{l}-3.77 \\
\end{array}$ & & & 1.16 & & & 1.78 & & & 6.64 & & & 6.54 & \\
\hline \multicolumn{21}{|l|}{ Europe } \\
\hline $\mathrm{BEO}$ & $\begin{array}{l}-1.91 \\
\end{array}$ & & & -4.70 & & & & & & & & & -0.60 & & & -4.52 & & & & \\
\hline BIR & $\begin{array}{l}-1.75 \\
\end{array}$ & & & -140.52 & & & -65.36 & & & 0.06 & & & -0.31 & & & -10.06 & & & & \\
\hline$\overline{C M N}$ & & & & & & & -20.90 & & & & & & & & & & & & & \\
\hline FKL & & & & & & & 0.60 & -3.17 & & & & & & & & & & & & \\
\hline HPB & \begin{tabular}{|l|}
-1.75 \\
\end{tabular} & & & $\begin{array}{l}-4.70 \\
\end{array}$ & & & -6.87 & & & 0.52 & & & & & & & & & & \\
\hline HYY & -1.76 & & & \begin{tabular}{|l|}
-7.53 \\
\end{tabular} & & & -24.11 & & & 3.71 & & & 0.47 & & & 1.46 & 1.46 & & -20.84 & \\
\hline $\begin{array}{l}\text { IPR } \\
\end{array}$ & \begin{tabular}{|l|}
-3.31 \\
\end{tabular} & & & -6.65 & & & $\begin{array}{l}-2.68 \\
\end{array}$ & & & -6.45 & & & 0.53 & & & -0.45 & & & 4.25 & \\
\hline JFJ & -12.60 & -6.43 & -2.97 & -6.86 & -4.31 & -2.52 & -3.71 & -2.88 & & -3.60 & $\begin{array}{l}-1.62 \\
\end{array}$ & & & & & -3.34 & -1.16 & -0.03 & -23.61 & -0.57 \\
\hline KPS & 0.02 & & & $\begin{array}{l}-0.09 \\
\end{array}$ & & & $\begin{array}{l}-0.48 \\
\end{array}$ & & & 1.34 & & & -0.10 & & & $\begin{array}{l}-0.49 \\
\end{array}$ & & & & \\
\hline MPZ & \begin{tabular}{|l|l|}
-1.33 \\
\end{tabular} & & & \begin{tabular}{|l|}
-2.60 \\
\end{tabular} & & & $\begin{array}{l}-4.56 \\
\end{array}$ & & & 1.48 & & & -0.21 & & & 0.11 & & & & \\
\hline MSY & \begin{tabular}{|l|}
-2.18 \\
\end{tabular} & & & \begin{tabular}{|l|}
-3.02 \\
\end{tabular} & & & $\begin{array}{l}-2.05 \\
\end{array}$ & & & $\begin{array}{l}-5.73 \\
\end{array}$ & & & 1.80 & & & 9.04 & & & & \\
\hline PAL & 2.51 & 0.79 & & 8.64 & 2.12 & & -1.04 & & & 5.55 & & & 0.00 & -0.04 & & -2.82 & -1.20 & & & \\
\hline PAY & & & & & & & -1.54 & & & & & & & & & & & & & \\
\hline PUY & -0.83 & & & -1.92 & & & $\bar{l}-\overline{1813.71}$ & & & 2.15 & & & 0.59 & & & -2.69 & & & & \\
\hline UGR & $\begin{array}{l}-0.17 \\
\end{array}$ & & & 0.11 & & & $\begin{array}{l}-0.68 \\
\end{array}$ & & & 1.24 & & & 0.62 & & & 1.08 & & & & \\
\hline ZSF & & & & & & & -23.63 & & & & & & & & & & & & & \\
\hline \multicolumn{21}{|c|}{ North America } \\
\hline ACA & -2.21 & -1.45 & -1.01 & & & & & & & & & & & & & & & & & \\
\hline
\end{tabular}




\begin{tabular}{|c|c|c|c|c|c|c|c|c|c|c|c|c|c|c|c|c|c|c|c|}
\hline APP & -1.58 & & & $\begin{array}{l}-2.89 \\
\end{array}$ & & & -7.44 & & & 1.13 & & & 0.80 & & & -0.82 & & & 14.80 \\
\hline BND & -1.47 & -1.04 & -0.72 & -3.35 & -2.13 & -1.49 & -3.63 & -3.42 & -1.37 & -1.93 & -0.30 & -0.39 & 0.22 & 0.30 & 0.10 & 0.71 & 0.13 & 0.46 & \\
\hline CPR & 0.78 & & & 2.42 & & & -11.68 & & & 6.25 & & & -0.06 & & & -10.55 & & & 19.42 \\
\hline EGB & -0.06 & & & 3.16 & & & 15.18 & & & -0.52 & & & 1.56 & & & -3.25 & -3.00 & & \\
\hline GBN & -4.21 & & & & & & & & & & & & & & & & & & \\
\hline GLR & 0.58 & & & & & & & & & & & & & & & & & & \\
\hline HGC & $\begin{array}{l}-3.64 \\
\end{array}$ & -2.76 & -1.36 & & & & & & & & & & & & & & & & \\
\hline MCN & -1.54 & -1.36 & -1.04 & & & & & & & & & & & & & & & & \\
\hline MRN & -0.03 & -0.32 & -0.59 & & & & & & & & & & & & & & & & \\
\hline RMN & -3.04 & & & & & & & & & & & & & & & & & & \\
\hline SGP & -1.04 & -0.86 & -0.58 & -2.30 & -2.10 & -1.10 & -2.06 & & & -1.17 & & & 0.56 & 0.17 & 0.32 & -1.32 & -1.35 & -1.06 & 7.01 \\
\hline SHN & -1.88 & -1.68 & -1.16 & & & & & & & & & & & & & & & & \\
\hline THD & $\begin{array}{l}-1.99 \\
\end{array}$ & & & $\begin{array}{l}-6.43 \\
\end{array}$ & & & -4.95 & & & -1.44 & & & 0.36 & & & 19.33 & & & 3.87 \\
\hline \multicolumn{20}{|c|}{ South Pacific } \\
\hline CGO & 2.21 & & & & & & -0.59 & & & 5.51 & & & & & & & & & \\
\hline MLO & -32.28 & 2.54 & 6.45 & -0.08 & 0.90 & & -2.81 & 1.72 & & 3.59 & -3.58 & & 1.75 & 0.60 & & -3.00 & -8.07 & -4.40 & 26.87 \\
\hline \multicolumn{20}{|c|}{ Polar regions } \\
\hline ALT & -2.57 & & & -3.13 & & & -1.93 & & & 1.25 & & & 0.86 & & & 3.42 & & & 9.69 \\
\hline BRW & -3.32 & 0.01 & 0.21 & -10.06 & 1.93 & 2.75 & $\begin{array}{l}-2.76 \\
\end{array}$ & 1.63 & 0.30 & 2.94 & 13.80 & 2.20 & 1.32 & 0.47 & 0.20 & 1.11 & -9.69 & -22.49 & 5.28 \\
\hline NMY & 3.73 & & & 0.18 & & & -0.81 & & & 2.94 & & & -0.86 & & & -45.17 & & & \\
\hline SPO & 1.55 & -0.27 & 2.87 & 3.17 & 0.22 & & & & & & & & 3.69 & 0.29 & & -5.89 & -5.46 & -4.99 & \\
\hline SUM & & & & & & & -0.61 & -0.58 & & & & & & & & & & & \\
\hline TIK & & & & & & & -14.66 & & & & & & & & & & & & -4.37 \\
\hline ZEP & 5.72 & & & 4.75 & & & 0.39 & & & 3.49 & & & 0.22 & 0.22 & & -3.65 & -1.11 & -3.65 & 0.78 \\
\hline
\end{tabular}


4) Evolution of $10 y$ trends

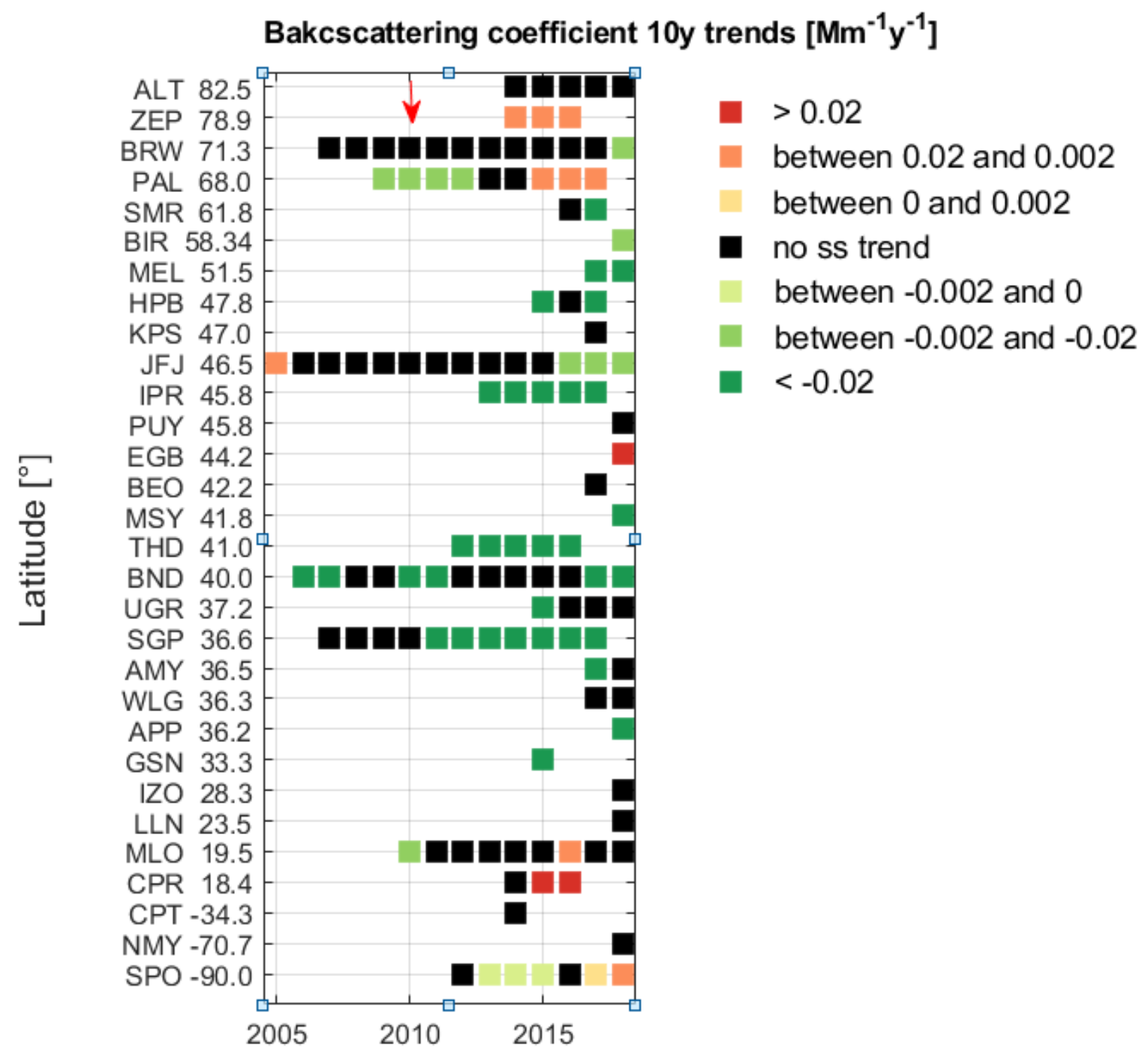

Fig. S7: Time series of $10 \mathrm{y}$ backscattering coefficient trends as a function of station latitude.

5) $\mathrm{RH}$ trends

Table S4: MK present-day trends in \%/y of $\mathrm{RH}$ measured by the nephelometer. The ss trends are given in bold.

\begin{tabular}{|c|c|c|c|}
\hline & MK 10y & MK 20y & MK 30y \\
\hline ACA & 0.00 & 0.04 & 0.04 \\
\hline APP & $-\mathbf{0 . 3 4}$ & $-\mathbf{0 . 3 3}$ & \\
\hline BND & -0.08 & 0.11 & $\mathbf{0 . 1 1}$ \\
\hline CPR & $\mathbf{0 . 0 8}$ & & \\
\hline
\end{tabular}




\begin{tabular}{|c|c|c|c|}
\hline EGB & -0.51 & & \\
\hline GBN & -0.25 & & \\
\hline GLR & -0.03 & & \\
\hline MCN & -0.06 & 0.02 & 0.06 \\
\hline MRN & $\mathbf{0 . 0 8}$ & 0.00 & -0.03 \\
\hline RMN & 0.04 & & \\
\hline SHN & $\mathbf{0 . 3 1}$ & $\mathbf{0 . 1 5}$ & $\mathbf{0 . 1 3}$ \\
\hline THD & $-\mathbf{0 . 3 0}$ & & \\
\hline HGC & -0.18 & -0.12 & -0.02 \\
\hline BEO & -0.23 & & \\
\hline BIR & $-\mathbf{0 . 1 3}$ & & \\
\hline HPB & 0.03 & & \\
\hline HYY & $\mathbf{0 . 1 2}$ & & \\
\hline IPR & -0.17 & & \\
\hline MSY & -0.18 & & \\
\hline PAL & 0.14 & 0.09 & \\
\hline PUY & $\mathbf{0 . 3 0}$ & & \\
\hline UGR & $\mathbf{- 0 . 5 5}$ & & \\
\hline AMY & $\mathbf{0 . 5 6}$ & & \\
\hline LLN & $\mathbf{- 1 . 8 0}$ & $\mathbf{- 1 . 8 8}$ & \\
\hline ALT & 0.00 & & \\
\hline BRW & 0.04 & $\mathbf{0 . 0 5}$ & \\
\hline IZO & $\mathbf{- 0 . 7 2}$ & & \\
\hline CGO & -0.02 & & \\
\hline & & & \\
\hline
\end{tabular}

6) Relation between SSA trends and absorption and scattering trends

The SSA trends depend directly on $\sigma_{\mathrm{sp}}$ and $\sigma_{\mathrm{ap}}$ trends. A $\sigma_{\mathrm{ap}}$ trend expressed in $\% / \mathrm{y}$ larger (lower) than the $\sigma_{\mathrm{sp}}$ trend will result in a decreasing (increasing) SSA trend, respectively (see Fig. S7). In contrast, when the $\sigma_{\mathrm{ap}}$ trend (in \%) is lower than the $\sigma_{\mathrm{sp}}$ trend an increasing SSA trend will result. The cyan and green curves in Fig. 7 give an idea of the variability of the SSA trend corresponding to Aethalometer measurement error (30\%) as well as to the potential $\mathrm{C}_{\text {ref }}$ errors for continental background aerosol (about $30 \%$ for $\mathrm{C}_{\text {ref }}$ between 2.5 and 4.5). The figure suggests that higher $\sigma_{\mathrm{ap}} / \sigma_{\mathrm{sp}}$ ratios lead to larger SSA trends for similar $\sigma_{\mathrm{ap}}$ and $\sigma_{\mathrm{sp}}$ trends. However, the simple rule still holds that when the $\sigma_{\mathrm{ap}}$ trend is larger than the $\sigma_{\mathrm{sp}}$ trend, the SSA trend will be decreasing (i.e., the aerosol will be getting darker). 


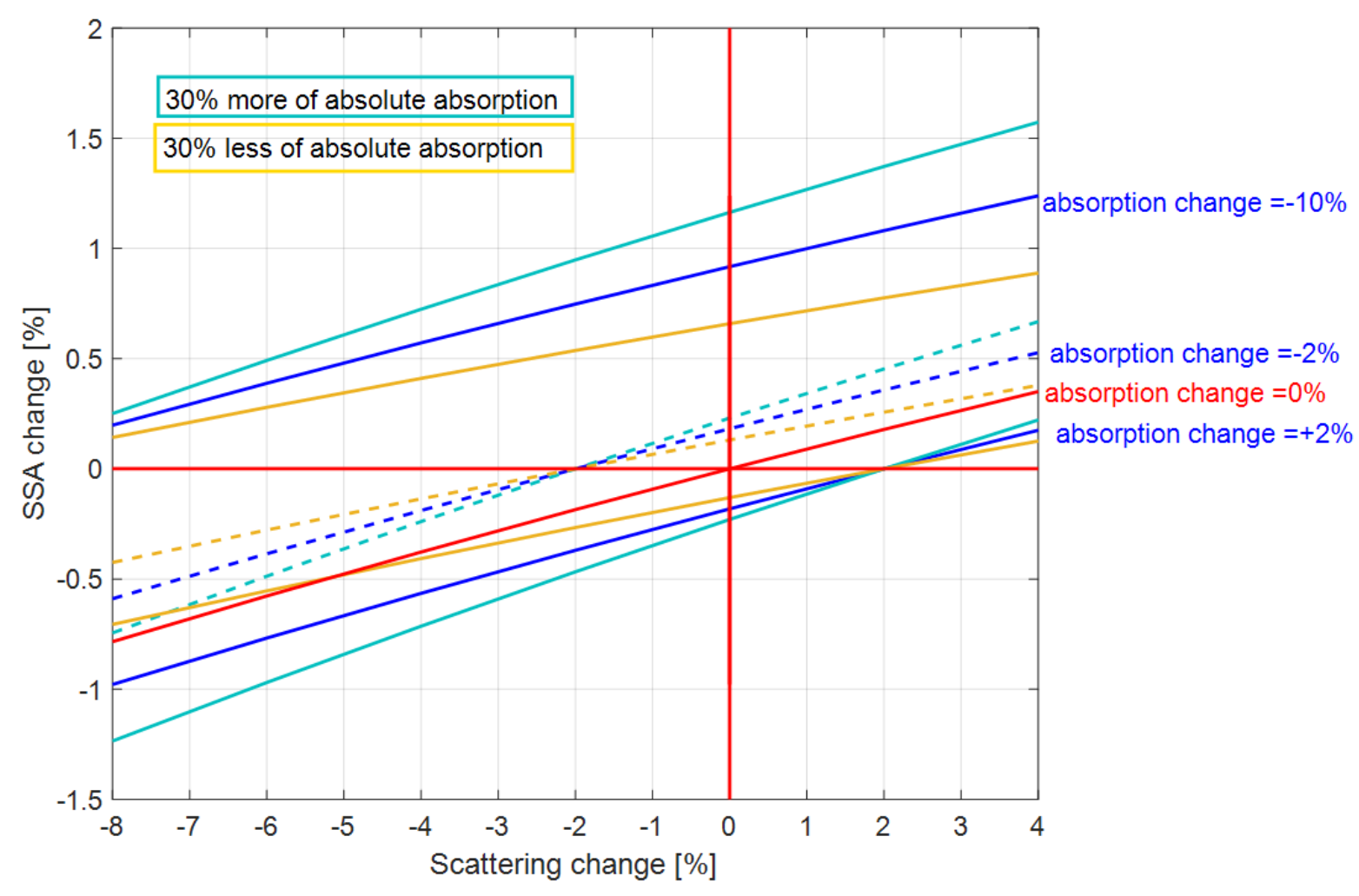

Figure S7: Single scattering albedo change as a function of the scattering coefficient change for several absorption coefficient changes. The change are always given in \%. The $\sigma_{\mathrm{ap}} / \sigma_{\mathrm{sp}}$ for the dark blue lines is of 0.1 . The green and cyan lines represent the same curves but for an increase of absorption of $+30 \%$ and $-30 \%$ respectively, corresponding to $\sigma_{\mathrm{ap}} / \sigma_{\mathrm{sp}}$ equal to 0.133 and 0.067 . The chosen $\sigma_{\mathrm{ap}}$ and $\sigma_{\mathrm{sp}}$ trend ranges correspond to the results of this study and the SSA trend range of this study is of $-1 \%$ to $0.4 \%$. 